\title{
Gauge field theory approach to spin transport in a 2D electron gas
}

\author{
B. Berche ${ }^{1,2}$, N. Bolívar ${ }^{3}$, A. López², E. Medina ${ }^{1,2}$ \\ ${ }^{1}$ Statistical Physics Group, P2M Department, Institut Jean Lamour, UMR CNRS 7198, BP 70239, F-54506 \\ Vandœuvre les Nancy Cedex, France \\ 2 Centro de Física, Instituto Venezolano de Investigaciones Científicas, Apartado 21827, Caracas 1020A, \\ Venezuela \\ 3 Departamento de Física, Universidad Central de Venezuela, Caracas, Venezuela
}

Received June 19, 2009

\begin{abstract}
We discuss the Pauli Hamiltonian including the spin-orbit interaction within an $U(1) \times S U(2)$ gauge theory interpretation, where the gauge symmetry appears to be broken. This interpretation offers new insight into the problem of spin currents in the condensed matter environment, and can be extended to Rashba and Dresselhaus spin-orbit interactions. We present a few outcomes of the present formulation: i) it automatically leads to zero spin conductivity, in contrast to predictions of Gauge symmetric treatments, ii) a topological quantization condition leading to voltage quantization follows, and iii) spin interferometers can be conceived in which, starting from an arbitrary incoming unpolarized spinor, it is always possible to construct a perfect spin filtering condition.
\end{abstract}

Key words: spin-orbit interaction, Gauge field theory, spin transport, spin Hall effect

PACS: $75.25 .+z, 85.75 .-d, 03.65 . \mathrm{Vf}$

\section{Introduction}

A reformulation of the spin-orbit (SO) coupling Hamiltonian in terms of non-Abelian gauge fields [1] was explicitly given in [2-5] where the SO interaction is presented as a $S U(2) \times U(1)$ gauge theory. As the Yang-Mills gauge theory is well understood and is the underpinning of well established theory, enormous insight can be brought upon new problems. Such gauge point of view, in more general terms, has been known for some time [6-9]. This formulation is very revealing, since the consistent gauge structure of the theory becomes obvious and the physics of spin currents, persistent currents and color diamagnetism [10] can be understood in a manner analogous to the well known $U(1)$ gauge theories. A consistent $S U(2) \times U(1)$ gauge approach was presented in reference $[4,5]$ where it was found that for the Pauli type Hamiltonians (including Rashba and two-dimensional reductions of the Dresselhaus Hamiltonian), Gauge Symmetry Breaking (GSB) is necessarily built into the theory and leads to the spin conductivity vanishing in constant electric fields [5]. In addition, the Yang Mills interpretation of the Rashba and Dresselhaus SO interactions renders the associated gauge fields real, with topological consequences analogous to the Aharonov Casher effect $[4,5]$.

The Rashba and Dresselhaus SO interactions arise in materials which lack either structural or bulk inversion symmetry, respectively [11-13]. These two kinds of interactions have recently been given a great deal of attention due to their potential role in the generation and manipulation of spin polarized currents, spin filters [14], spin accumulation [15], and spin optics [16].

\section{Spin-orbit interaction in semiconductors}

Spin-orbit interaction may be defined as the interaction of the spin magnetic moment of the electron, $\overrightarrow{\boldsymbol{\mu}}=-g \frac{\mathrm{e}}{2 m} \overrightarrow{\mathbf{s}}=-\frac{\mathrm{e} \hbar}{2 m} \overrightarrow{\boldsymbol{\sigma}}$ with the magnetic field produced by all external moving charges 
in the electron rest frame. Here, $\overrightarrow{\mathbf{s}}=\frac{1}{2} \hbar \overrightarrow{\boldsymbol{\sigma}}$ is the spin of the electron with $\overrightarrow{\boldsymbol{\sigma}}$ the vector of Pauli matrices, $g \simeq 2$ the Landé factor, $m$ the electron rest mass and - e the electron electric charge. Bold face symbols denote matrices in spin space. In the rest frame of the external charges, the SO interaction, properly corrected to take into account Thomas precession, is usually described by the Pauli term, $\sim \frac{1}{2 m^{2} c^{2}} \overrightarrow{\mathbf{s}} \cdot(\vec{p} \times \vec{\nabla} V)$, where $V$ is the potential energy of the electron in the presence of the external charges. For a spherically symmetric potential, $\vec{\nabla} V=f(r) \vec{r}$ and the SO interaction is proportional to $\overrightarrow{\mathrm{s}} \cdot \vec{L}$, hence its name.

In a semi-conductor, the electron rest mass and the Landé factor must be replaced by their effective counterparts, and the spin-orbit interaction can thus be considerably enhanced. Moreover, an appropriate basis in a crystalline solid is given in terms of the Bloch wave functions, $\psi(\vec{r})=$ $u_{\vec{k}} \mathrm{e}^{\mathrm{i} \vec{k} \vec{r}}$, and the SO interaction must be calculated in this basis. This is generally a hard task and one usually uses phenomenological expressions compatible with the crystal symmetries. When there is bulk inversion asymmetry (BIA), we have the Dresselhaus expression in $3 d$ systems [17],

$$
H_{D, 3 d}=\text { const. } k_{x}\left(k_{y}^{2}-k_{z}^{2}\right) \boldsymbol{\sigma}_{x}+\text { c.p. }
$$

where c.p. stands for cyclic permutations. When the electrons are confined in two dimensions, the expectation value along the third dimension should be considered, with $\left\langle k_{z}\right\rangle \simeq 0,\left\langle k_{z}^{2}\right\rangle \simeq(\pi / a)^{2}$, $a$ being the typical confinement length in the $z$-direction. The Dresselhaus SO interaction thus takes the simple form

$$
H_{D, 2 d}=\beta\left(k_{x} \boldsymbol{\sigma}_{x}-k_{y} \boldsymbol{\sigma}_{y}\right),
$$

where we neglect cubic terms in $k$. If the confining potential is not symmetric i. e. in the case of space inversion asymmetry (SIA), there is another term which directly follows from the expression $\frac{\hbar^{2}}{4 m^{2} c^{2}} \vec{\sigma} \cdot(\vec{k} \times \vec{\nabla} V)$ with only the $\left\langle\nabla_{z} V\right\rangle$ contribution. This term is known as the Rashba SO interaction,

$$
H_{R, 2 d}=\alpha\left(k_{y} \boldsymbol{\sigma}_{x}-k_{x} \boldsymbol{\sigma}_{y}\right)
$$

Note that the Rashba SO amplitude can be tuned experimentally using a gate voltage, since the coefficient $\alpha$ is proportional to the electric field. For more details on SO interactions in semiconductors, see $[13,17]$.

\section{Non-Abelian gauge field theory approach}

Let us first consider the Pauli SO interaction. Rashba and Dresselhaus SO interactions will be treated later. Neglecting electron-electron interactions, we start by considering the Pauli Hamiltonian to order $v / c$, acting on two-component spinors,

$$
\begin{aligned}
H= & {\left[\frac{(\vec{p}-\mathrm{e} \vec{A})^{2}}{2 m}+V-\mathrm{e} \phi\right] \mathbf{1}_{2 \times 2}-\left[\frac{\vec{p}^{4}}{8 m^{3} c^{2}}+\frac{\mathrm{e} \hbar^{2}}{8 m^{2} c^{2}} \nabla \cdot \vec{E}\right] \mathbf{1}_{2 \times 2} } \\
& +\frac{\mathrm{e} \hbar}{2 m} \overrightarrow{\boldsymbol{\sigma}} \cdot \vec{B}-\frac{\mathrm{e} \hbar \overrightarrow{\boldsymbol{\sigma}} \cdot(\vec{p}-\mathrm{e} \vec{A}) \times \vec{E}}{4 m^{2} c^{2}}
\end{aligned}
$$

where the first term in the first line corresponds to the usual Schrödinger equation including the kinetic energy with a minimal coupling to the electromagnetic field, the substrate potential denoted by $V$, that can be assumed periodic, and a scalar potential contribution. The second term in the first line describes the first relativistic correction to the kinetic energy and the Darwin term, where $\vec{E}$ is the electric field and $c$ the speed of light. These first two terms are proportional to the $2 \times 2$ identity matrix in spin space. The second line comprises explicitly spin-dependent terms, first the Zeeman interaction where $\vec{B}$ is the magnetic field and $\vec{\sigma}$ is the Pauli matrix vector and the second term is the spin-orbit interaction, now written with the minimal coupling to the gauge vector. We have assumed a static potential so that the rotor of the electric field is absent and the spin-orbit interaction is limited to the term mentioned here. In what follows, we absorb the spin-independent 
one-body interactions (second term of first line in equation (4)) in the potential, and we rewrite the non-relativistic kinetic energy plus the spin-orbit interaction as

$$
\begin{aligned}
\text { K.E. }+ \text { S.O. } & =\frac{1}{2 m}\left[(\vec{p}-\mathrm{e} \vec{A})^{2} \mathbf{1}_{2 \times 2}-2(\vec{p}-\mathrm{e} \vec{A}) \mathbf{1}_{2 \times 2} \frac{-\mathrm{e} \hbar}{4 m c^{2}} \overrightarrow{\boldsymbol{\sigma}} \times \vec{E}\right] \\
& =\frac{1}{2 m}\left[(\vec{p}-\mathrm{e} \vec{A})^{2} \mathbf{1}_{2 \times 2}-\frac{-\mathrm{e} \hbar}{4 m c^{2}} \overrightarrow{\boldsymbol{\sigma}} \times \vec{E}\right]^{2}-\frac{\mathrm{e}^{2} \hbar^{2}}{32 m^{3} c^{4}}|\overrightarrow{\boldsymbol{\sigma}} \times \vec{E}|^{2} .
\end{aligned}
$$

This suggests an $S U(2) \times U(1)$ form described by non-Abelian $W^{\mu a}$ and ordinary Abelian gauge fields $A^{\mu}=\left(A^{0}, A^{i}\right)=(\phi / c, \vec{A})$ with $\vec{E}=-\vec{\nabla} \phi-\partial_{t} \vec{A}$ and $\vec{B}=\vec{\nabla} \times \vec{A}$, and we can rewrite the Hamiltonian, following Jin, Li and Zhang [18] as

$$
\begin{aligned}
H= & \frac{1}{2 m}\left[(\vec{p}-\mathrm{e} \vec{A}) \mathbf{1}_{2 \times 2}-g \vec{W}^{a} \boldsymbol{\tau}^{a}\right]^{2} \\
& -\frac{1}{8 m} g^{2} \vec{W}^{a} \vec{W}^{a} \mathbf{1}_{2 \times 2}-g c W^{0 a} \boldsymbol{\tau}^{a}+\left(V^{\prime}-\mathrm{e} c A^{0}\right) \mathbf{1}_{2 \times 2},
\end{aligned}
$$

where the Zeeman interaction is written as the time component of the non-Abelian gauge field [18] $-g c W^{0 a} \boldsymbol{\tau}^{a}=\frac{\mathrm{e} \hbar}{2 m} \overrightarrow{\boldsymbol{\sigma}} \cdot \vec{B}$, while the space components of this $S U(2)$ connection are defined by $g W^{i a} \boldsymbol{\tau}^{a}=-\left(\mathrm{e} \hbar / 2 m c^{2}\right) \varepsilon_{i a j} E^{j} \boldsymbol{\tau}^{a}$, or explicitly,

$$
\begin{aligned}
g \vec{W}^{1} & =\frac{\mathrm{e} \hbar}{2 m c^{2}}\left(E_{z} \vec{u}_{y}-E_{y} \vec{u}_{z}\right) \\
g \vec{W}^{2} & =\frac{\mathrm{e} \hbar}{2 m c^{2}}\left(-E_{z} \vec{u}_{x}+E_{x} \vec{u}_{z}\right) \\
g \vec{W}^{3} & =\frac{\mathrm{e} \hbar}{2 m c^{2}}\left(E_{y} \vec{u}_{x}-E_{x} \vec{u}_{y}\right) .
\end{aligned}
$$

with $\vec{u}_{x}, \vec{u}_{y}$ and $\vec{u}_{z}$ unit vectors in the $x-, y-$ and $z$-directions. The $2 \times 2$ matrices $\tau^{a}$ are the symmetry generators for $S U(2)$ obeying the commutation relation $\left[\boldsymbol{\tau}^{a}, \boldsymbol{\tau}^{b}\right]=\mathrm{i} \varepsilon_{a b c} \boldsymbol{\tau}^{c}, \varepsilon_{a b c}$ being the totally antisymmetric tensor. The coupling constant $g$ is fixed by the combination $g W^{\mu a}$ which has the dimensions of $\frac{\mathrm{e} \hbar}{2 m c^{2}} E$, and we choose $g=\hbar$. The relation between the spin operator and the corresponding generators is $\hbar \boldsymbol{\tau}^{a}=\mathbf{s}^{a}$ and the spin is $s=1 / 2$. The superscripts of the beginning of the Latin alphabet, $a, b, c, \ldots$ refer to the internal spin degrees of freedom for which we use the convention of summation when they are repeated, while Greek indices $\mu, \nu, \ldots$ correspond to space-time components and run from 0 to 3 , the time component corresponding to 0 and the space components being also denoted as Latin indices from the middle of the alphabet, $i, j, k, \ldots$

This formulation differs from that of $[10,18]$ most importantly in the term quadratic in $\vec{W}^{a}$ in equation (6). The purpose of writing the second term here, as a function of the $S U(2)$ connection is to evidence gauge symmetry breaking (GSB) in this Hamiltonian. This observation has important consequences in the physical interpretation of the resulting Yang-Mills fields and is the reason why the Yang-Mills fields themselves are observable quantities, whereas in a gauge symmetric theory they would be gauge dependent $[19,20]$.

In order to generate the Noether currents in a canonical fashion, one must formulate the appropriate Lagrangian for the corresponding equations of motion. The non-relativistic Lagrangian density we seek is (now omitting identity matrices)

$$
\begin{aligned}
\mathcal{L}= & \frac{\mathrm{i} \hbar}{2}\left(\Psi^{\dagger} \dot{\Psi}-\dot{\Psi}^{\dagger} \Psi\right)-\frac{1}{2 m}(-\mathrm{i} \hbar \overrightarrow{\mathcal{D}} \Psi)^{\dagger}(-\mathrm{i} \hbar \overrightarrow{\mathcal{D}} \Psi) \\
& -\Psi^{\dagger}\left(\frac{-g^{2}}{8 m} \vec{W}^{b} \vec{W}^{b}+g c W^{0 a} \boldsymbol{\tau}^{a}+\mathrm{ec} A^{0}\right) \Psi \\
& -\frac{\mathrm{e}^{2}}{4 m} F_{\mu \nu} F^{\mu \nu}-\frac{g^{2}}{4 m} G_{\mu \nu}^{a} G^{\mu \nu a},
\end{aligned}
$$

where $\Psi$ is a Pauli spinor $\Psi=\left(\begin{array}{c}\psi_{\uparrow} \\ \psi_{\downarrow}\end{array}\right), G_{\mu \nu}^{a}=\partial_{\mu} W_{\nu}^{a}-\partial_{\nu} W_{\mu}^{a}-\epsilon^{a b c} W_{\nu}^{b} W_{\mu}^{c}$ and $F_{\mu \nu}=\partial_{\mu} A_{\nu}-\partial_{\nu} A_{\mu}$ are the $S U(2)$ and $U(1)$ field tensors respectively. The new term $-\frac{g^{2}}{8 m} \Psi^{\dagger} \vec{W}^{b} \vec{W}^{b} \Psi$ is due to gauge 
symmetry breaking. The covariant derivative is then of the form $-\mathrm{i} \hbar \overrightarrow{\mathcal{D}}=-\mathrm{i} \hbar \vec{\nabla}-\mathrm{e} \vec{A}-g \vec{W}^{a} \boldsymbol{\tau}^{a}$. This form of the covariant derivative determines the well known $U(1)$ coupling constant $e / \hbar$ and the $S U(2)$ coupling constant for this theory is $g / \hbar$. A gauge transformation of the Lagrangian density would leave it unchanged up to a divergence at the condition that the Coulomb gauge for the $S U(2)$ connection is satisfied, $\vec{\nabla} \cdot \vec{W}^{a}=0$.

The Hamiltonian in equation (6) is derived from the corresponding Lagrange equations for the matter fields $\Psi$. The equations of motion of the Yang-Mills fields in the presence of currents (generalized Maxwell equations) follow from Euler-Lagrange equations with respect to variations of the gauge fields,

$$
\partial_{\mu} \frac{\partial \mathcal{L}}{\partial\left(\partial_{\mu} W_{\nu}^{a}\right)}=\frac{\partial \mathcal{L}}{\partial W_{\nu}^{a}}
$$

The 1.h.s. of this equation gives $\partial_{\mu} \frac{\partial \mathcal{L}}{\partial\left(\partial_{\mu} W_{\nu}^{a}\right)}=-\partial_{\mu} G^{\mu \nu a}$, and since the non-Abelian field tensor $G^{\mu \nu a}$ is antisymmetric with respect to space-time indices, it is natural to introduce a conserved current $\mathcal{J}^{\nu a}=\frac{\partial \mathcal{L}}{\partial W_{\nu}^{a}}$. The conserved current $\mathcal{J}^{\nu a}[19]$ is the full spin current carried both by matter and radiation i. e. $\overrightarrow{\mathcal{J}}^{a}=\overrightarrow{\mathcal{J}}_{\text {Matter }}^{a}+\overrightarrow{\mathcal{J}}_{\text {Radiation. }}^{a}$ This full current is an observable physical quantity, since the gauge is fixed in the present case (in contradistinction with ordinary $S U(2)$ gauge theory where it is gauge-dependent). The spatial (spin current) and time (magnetization) components of the current density then follow as

$$
\begin{aligned}
\overrightarrow{\mathcal{J}}^{a}= & \frac{g}{2 m}\left[\left(\boldsymbol{\tau}^{a} \Psi\right)^{\dagger}(-\mathrm{i} \hbar \overrightarrow{\mathcal{D}} \Psi)+(-\mathrm{i} \hbar \overrightarrow{\mathcal{D}} \Psi)^{\dagger}\left(\boldsymbol{\tau}^{a} \Psi\right)\right] \\
& +\Psi^{\dagger}\left(\frac{g^{2}}{4 m} \vec{W}^{a}\right) \Psi \\
& +\frac{g^{2}}{m} \varepsilon_{a b c} W_{\nu}^{b}\left(G^{\nu x c} \vec{u}_{x}+G^{\nu y c} \vec{u}_{y}+G^{\nu z c} \vec{u}_{z}\right),
\end{aligned}
$$

and the spin polarization

$$
\mathcal{J}^{0 a}=\Psi^{\dagger} g \boldsymbol{\tau}^{a} \Psi+\frac{g^{2}}{m} \varepsilon_{a b c} W_{j}^{b} G^{j 0 c} .
$$

Three terms can be distinguished in the spin current.

i) The first term has the canonical form expected for the material current namely

$$
\overrightarrow{\mathcal{J}}_{\text {Matter }}^{a}=(g / 2) \Psi^{\dagger}\left(\boldsymbol{\tau}^{a} \vec{v}+\vec{v} \boldsymbol{\tau}^{a}\right) \Psi
$$

where $v^{i}=(1 / \mathrm{i} \hbar)\left[r^{i}, H\right]$.

ii) The second term comes from the gauge symmetry breaking contribution. It depends on both matter and radiation.

iii) Finally, the third term is the canonical radiative contribution originating from the derivative with respect to the gauge potential of the non-Abelian contribution of the field tensor $G_{\mu \nu}^{a}$. Note that this last term would not arise in an Abelian theory (e. g. in $U(1)$, since the photon does not carry any electric charge).

The first and last terms were described in [18] as taken from an apparently gauge symmetric form. The magnetization term has both a material contribution (the first term) and a radiative contribution as both matter and radiation carry angular momentum. We emphasize that the extent to which gauge symmetry is broken depends on the choice of the electric field. If only the $E_{z} \neq 0$, then one allows gauge transformations that leave $W_{1}^{2}=-W_{2}^{1}$ invariant. This is analogous to the remnant $Z_{2}$ group after $U(1)$ GSB in superconductors and a similar situation in the electro-weak GSB mechanism [19]. 
Since the gauge theory considered here is non-Abelian, in the most general case, the corresponding current is not gauge invariant (as it would be in the $U(1)$ case i. e. electromagnetism) and thus a function that is not measurable [22]. An important property in the SO case is that the gauge field is determined by the physical electric field, and thus the gauge is fixed and the spin current becomes properly defined. The third term in equation (12), which has the structure of a gauge symmetry breaking term, would also by itself fix the gauge and it has further consequences to be discussed below.

Rewriting the last term in equation (12) in terms of ordinary derivatives plus a gauge dependent term we obtain

$$
-\frac{\mathrm{i} \hbar g}{2 m}\left[\left(\boldsymbol{\tau}^{a} \Psi\right)^{\dagger} \vec{\nabla} \Psi-(\vec{\nabla} \Psi)^{\dagger}\left(\boldsymbol{\tau}^{a} \Psi\right)\right]-\Psi^{\dagger}\left(\frac{g^{2}}{4 m} \vec{W}^{a}\right) \Psi .
$$

The second term is the non-Abelian analog of the London term in superconductivity. The main result of this approach is then to recognize that such second term exactly cancels the symmetry breaking term in equation (12) and renders zero matter currents proportional to the electric field (zero spin conductivity in arbitrary space dimension). The scenario is now very different from superconductivity: there, the London term is the only one remaining after symmetry breaking, while for the non-Abelian case, the London contribution gets cancelled. As discussed in references $[10,21]$, equilibrium currents remain in relation to the leftover radiative contribution, cubic in the non-Abelian potential plus a field independent matter contribution.

\section{Abelian analogy}

In order to discuss an analogy, we will consider the simpler case of the $U(1)$ Abelian gauge theory. Then, the Lagrangian density reads as [23]

$$
\mathcal{L}=\mathrm{i} \hbar \psi^{*} \partial_{t} \psi-\frac{1}{2 m}(-\mathrm{i} \hbar \overrightarrow{\mathcal{D}} \psi)^{*}(-\mathrm{i} \hbar \overrightarrow{\mathcal{D}} \psi)-\psi^{*}(\mathrm{e} \phi) \psi+\frac{1}{2 \mu_{0}} \vec{B}^{2}-\frac{1}{2} \varepsilon_{0} \vec{E}^{2}
$$

and minimization of the action with respect to the gauge field leads to

$$
\frac{\mathrm{i} \hbar \mathrm{e}}{2 m}\left(\psi \vec{\nabla} \psi^{*}-\psi^{*} \vec{\nabla} \psi\right)-\frac{\mathrm{e}^{2}}{m} \vec{A} \psi^{*} \psi-\frac{1}{\mu_{0}} \vec{\nabla} \times \vec{B}=0
$$

from which the charge current density follows

$$
\vec{j}=\frac{\mathrm{e}}{2 m}\left(\psi^{*}(-\mathrm{i} \hbar \vec{\nabla} \psi)+(-\mathrm{i} \hbar \vec{\nabla} \psi)^{*} \psi\right)-\frac{\mathrm{e}^{2}}{m} \vec{A} \psi^{*} \psi
$$

The first term is usually referred to as the paramagnetic term, while the second term is responsible for diamagnetic properties of matter. If one had changed the Lagrangian in equation (16), adding a GSB term as

$$
\mathcal{L} \longrightarrow \mathcal{L}+\frac{\mathrm{e}^{2}}{2 m} \vec{A}^{2} \psi^{*} \psi
$$

the current would have changed to its paramagnetic contribution only, $i$. $e$. without any dependence on the gauge field (no second term in the r.h.s. of equation (18)). This is what occurs, as a first approximation, in a paramagnetic metal in a weak magnetic field. The cancellation of this diamagnetic term corresponds to the similar scenario of any spin Hall effect vanishing in the Pauli SO case.

\section{Some implications}

\subsection{Vanishing of the spin Hall conductivity for Rashba materials in arbitrary dimension}

As we have mentioned, the presence of the gauge symmetry breaking term in the Lagrangian density exactly compensates the "diamagnetic" (also called diacolor) term in the spin current 
density, and therefore there cannot be any spin current proportional to the electric field [5]. This means that the spin Hall conductivity identically vanishes, and this result is true in arbitrary dimensions. In the particular case of a two-dimensional system with Rashba SO interaction, Rashba has shown, using sum rule arguments, that there is no spin Hall conductivity [24]. Our conclusion is more general in the sense that in any dimension we obtain a spin Hall conductivity which vanishes due to an exact cancellation between two terms. In two dimensions, the situation is special in the sense that both terms do vanish!

\subsection{Voltage quantization}

We now consider a two-dimensional electron gas (2DEG) in a crystal, and we analyze the consequence of periodicity in the real space. If we consider the transport of the spinor [25] along the primitive cell of the crystal, we have to form the quantities like

$$
\exp \left(\mathrm{i} \hbar^{-1} \oint\left(\vec{p}+g \vec{W}^{a} \boldsymbol{\tau}^{a}\right) \mathrm{d} \vec{r}\right),
$$

in the absence of magnetic field. If we restrict ourselves to the transport along vectors $\vec{a}$ and $\vec{b}$ (or along $\vec{b}$, then along $\vec{a}$ ) in a uniform external electric field, the commutator $\left[T_{\vec{a}}, T_{\vec{b}}\right]$ should be considered, where $T_{\vec{a}}=\exp \left(\mathrm{i} \hbar^{-1}\left(\vec{p}+g \vec{W}^{a} \tau^{a}\right) \vec{a}\right)$. This commutator can be shown to be proportional to $\sin \frac{\mathrm{e}}{2 m c^{2}}|\vec{a} \times \vec{E}| \sin \frac{\mathrm{e}}{2 m c^{2}}|\vec{b} \times \vec{E}|$, thus $\left[T_{\vec{a}}, T_{\vec{b}}\right]$ vanishes when $\vec{E}$ is in the plane of the 2DEG [5]. On the other hand, when $\vec{E}$ is perpendicular to the plane, a quantization condition appears for the voltage along at least an arm of the elementary cell, say the arm $a$

$$
E_{z} a=p \pi m c^{2} / \mathrm{e}
$$

with $p$ an integer. The quantity $2 \pi /\left(2 m c^{2} / \mathrm{e}\right)$ plays the role of a quantum of voltage similar to the flux quantum in the Aharonov-Bohm (AB) effect [5]. The analogy is nevertheless not complete, and there are important differences. While the gauge invariant phase in the $\mathrm{AB}$ effect is given by $\oint \vec{A} \mathrm{~d} \vec{r}$ along a closed path, in the present situation the corresponding quantity reduces to the integral along an open path, $\int|\vec{E} \times \mathrm{d} \vec{r}|$. This is, of course, due to the fact that the non-Abelian gauge field is given by the electric field itself, which is gauge invariant already (and there is no need to close the path to render the phase gauge invariant). The present case is thus more like the Aharonov-Casher effect [26].

Let us mention that in a semi-conductor, using the effective mass of the electron instead of its bare mass, the quantum of voltage would be considerably reduced.

\subsection{Spin interferometry}

In this section we consider an electronic Mach-Zehnder interferometer where electron beams can interfere and are then collected in two distinct detectors [27]. A magnetic field perpendicular to the plane of the interferometer creates a gauge vector $\vec{A}$ in the whole space. The magnetic field could be limited to a narrow area and does not "touch" the arms of the interferometer. In the illustrative case below we consider that the electron mirrors and beam splitters, realized by gate potentials, are diagonal in spin space, i.e. they do not mix spin components. Consideration of the changes in the electron propagation direction on the spin components $[28,31]$ does not change the qualitative scenario drawn below.

Let us first consider the Aharonov-Bohm situation where the spin of the electrons is neglected, and we only discuss the case of one detector. The electrons can follow one of the two paths called I and II in figure 1. The two electron paths are supposed to be essentially one-dimensional.

The wave function, transported along each path to detector $D_{a}$ reads as

$$
\begin{aligned}
\psi_{a}= & r_{2} \exp \left(\mathrm{i} \hbar^{-1} \int_{I^{\prime}}(\vec{p}+\mathrm{e} \vec{A}) \mathrm{d} \vec{r}\right) \exp \left(\mathrm{i} \hbar^{-1} \int_{I}(\vec{p}+\mathrm{e} \vec{A}) \mathrm{d} \vec{r}\right) t_{1} \\
& +t_{2} \exp \left(\mathrm{i} \hbar^{-1} \int_{I I^{\prime}}(\vec{p}+\mathrm{e} \vec{A}) \mathrm{d} \vec{r}\right) \exp \left(\mathrm{i} \hbar^{-1} \int_{I I}(\vec{p}+\mathrm{e} \vec{A}) \mathbf{r} \mathrm{d} \vec{r}\right) r_{1} \Psi_{0} .
\end{aligned}
$$




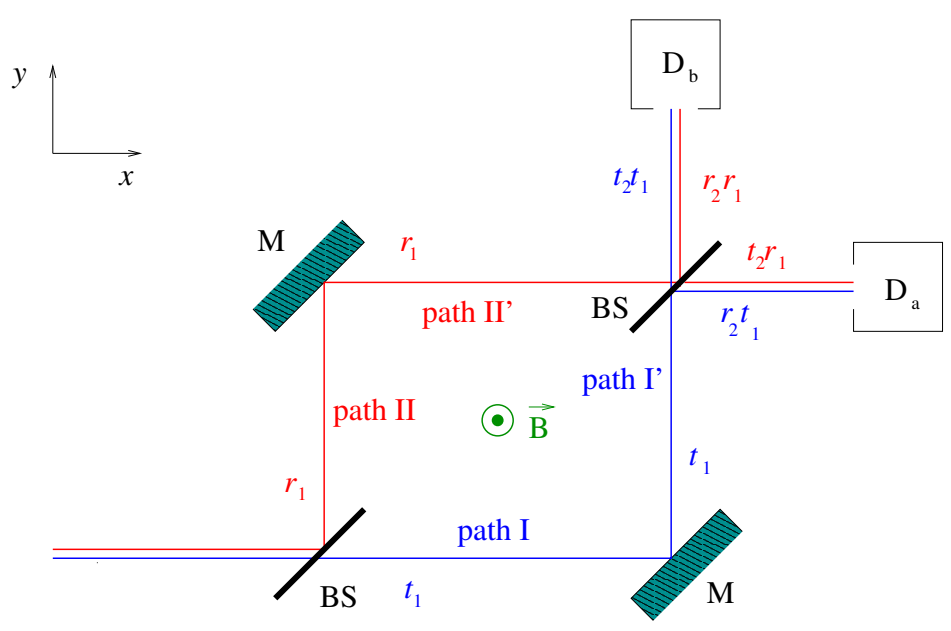

Figure 1. Mach-Zehnder electron interferometer: an electron beam is first split into two distinct beams through a Beam Splitter (BS), each of the beams is then reflected on a Mirror (M) before being collected at another BS. Two detectors (D) can measure the beam intensity in two perpendicular directions. An external magnetic field is applied in the perpendicular direction.

In order to calculate the probability amplitude in the detector, we have now to evaluate the quantities like

$$
\exp \left(\mathrm{i} \hbar^{-1} \int_{I^{\prime}} \Pi_{y} \mathrm{~d} y\right) \exp \left(\mathrm{i} \hbar^{-1} \int_{I} \Pi_{x} \mathrm{~d} x\right)
$$

with $\pi_{i}=p_{i}+\mathrm{e} A_{i}$ (take care to the non standard definition here, in order to shorten the expression of the comparator operator [22]). Using the Baker-Campbell-Hausdorff (BCH) formula and the fact that the commutator $\left[\pi_{x}, \pi_{y}\right]$ is a $c$-number, this is equivalent to

$$
\exp \left(\mathrm{i} \hbar^{-1}\left(\int_{I^{\prime}} \Pi_{y} \mathrm{~d} y+\int_{I} \Pi_{x} \mathrm{~d} x\right)-\frac{1}{2}\left(\mathrm{i} \hbar^{-1}\right)^{2} \int_{I^{\prime}} \mathrm{d} y \int_{I} \mathrm{~d} x\left[\Pi_{y}, \Pi_{x}\right]\right) .
$$

The interferences are due to the last term involving $\left[\Pi_{y}, \Pi_{x}\right]=\left[p_{y}+\mathrm{e} A_{y}, p_{x}+\mathrm{e} A_{x}\right]=\mathrm{i} \hbar \mathrm{e} B$, so the last integral contributes to the phase by an amount $\mathrm{i} \pi \Phi_{\mathrm{B}} / \Phi_{0}$ with $\Phi_{0}=h /|\mathrm{e}|$ the quantum of magnetic flux (associated with the charge e). Eventually, one has

$$
\psi_{a}=\left(r_{2} t_{1} \mathrm{e}^{\mathrm{i} \pi \Phi_{\mathrm{B}} / \Phi_{0}}+t_{2} r_{1} \mathrm{e}^{-\mathrm{i} \pi \Phi_{\mathrm{B}} / \Phi_{0}}\right) \mathrm{e}^{\mathrm{i} \alpha} \psi_{0},
$$

where the phase $\alpha$ comes from the non-interfering contribution. This result is the standard AharonovBohm effect which states that the interference pattern is determined by the total magnetic flux enclosed between the two arms of the interferometer.

We now consider a variant of this problem where the spin-orbit interaction is taken into account. The arms of the interferometer are supposed to be made of a "Rashba-Dresselhaus" active medium [28].

Instead of transporting a wave function with a phase variation induced by the coupling of the electron charge to the Abelian gauge vector, we now consider the transport of a Pauli spinor and its precession due to the coupling of the electron spin to the non-Abelian gauge field. In the case when both Rashba and Dresselhaus SO interactions are present, the Hamiltonian is given by the following expression $[29,30]$,

$$
H=\frac{\vec{\pi}^{2}}{2 m}+V+\alpha\left(\pi_{x} \boldsymbol{\sigma}^{y}-\pi_{y} \boldsymbol{\sigma}^{x}\right)+\beta\left(\pi_{y} \boldsymbol{\sigma}^{y}-\pi_{x} \boldsymbol{\sigma}^{x}\right),
$$

where $\vec{\pi}=\vec{p}-\mathrm{e} \vec{A}$. The non-Abelian gauge field now takes the form

$$
g \vec{W}^{a} \boldsymbol{\tau}^{a}=\left(\beta \boldsymbol{\tau}^{x}-\alpha \boldsymbol{\tau}^{y}\right) \vec{u}_{x}+\left(\alpha \boldsymbol{\tau}^{x}-\beta \boldsymbol{\tau}^{y}\right) \vec{u}_{y}
$$


and the spinor transport along the paths of the interferometer is defined by the operator

$$
\Psi_{a}=\mathcal{U}_{a} \Psi_{0},
$$

according to

$$
\begin{aligned}
\mathcal{U}_{a}= & r_{2} \exp \left(\mathrm{i} \hbar^{-1} \int_{I^{\prime}}\left(\vec{\Pi}+g \vec{W}^{a} \boldsymbol{\tau}^{a}\right) \mathrm{d} \vec{r}\right) \exp \left(\mathrm{i} \hbar^{-1} \int_{I}\left(\vec{\Pi}+g \vec{W}^{a} \boldsymbol{\tau}^{a}\right) \mathrm{d} \vec{r}\right) t_{1} \\
& +t_{2} \exp \left(\mathrm{i} \hbar^{-1} \int_{I I^{\prime}}\left(\vec{\Pi}+g \vec{W}^{a} \boldsymbol{\tau}^{a}\right) \mathrm{d} \vec{r}\right) \exp \left(\mathrm{i} \hbar^{-1} \int_{I I}\left(\vec{\Pi}+g \vec{W}^{a} \boldsymbol{\tau}^{a}\right) \mathrm{d} \vec{r}\right) r_{1}
\end{aligned}
$$

Again, the evaluation of the previous expressions is made delicate due to non-commutativity of the gauge field components (and their commutator is no longer a simple $c$-number), so the $\mathrm{BCH}$ formula is now of no use, since it would require the complete nested expression. We rather use properties of the Pauli matrices to decompose the exponential functions, $\exp \left( \pm \mathrm{i} \gamma \boldsymbol{\sigma}^{n}\right)=\cos \gamma \pm$ $\mathrm{i} \boldsymbol{\sigma}^{n} \sin \gamma$. After some algebra, we get

$$
\mathcal{U}_{a}=\mathcal{A}_{+}\left[\cos ^{2} \Lambda-\sin ^{2} \Lambda \sin 2 \theta\right] \mathbf{1}_{2 \times 2}+\mathrm{i}(\sin \Lambda) \mathbf{M}_{2 \times 2},
$$

where we have introduced the traceless matrix

$$
\mathbf{I}_{2 \times 2}=\mathcal{A}_{-}(\sin \Lambda \cos 2 \theta) \boldsymbol{\sigma}^{z}-\mathcal{A}_{+} \cos \Lambda(\cos \theta+\sin \theta)\left(\boldsymbol{\sigma}^{x}-\boldsymbol{\sigma}^{y}\right),
$$

the dimensionless variables

$$
\begin{gathered}
\Lambda=\left(m^{*} L / \hbar\right) \sqrt{\alpha^{2}+\beta^{2}}, \\
\theta \equiv \tan ^{-1}(\beta / \alpha),
\end{gathered}
$$

and the coefficients

$$
\mathcal{A}_{ \pm}=t_{1} t_{2} \pm r_{1} r_{2} \mathrm{e}^{2 \mathrm{i} \pi \Phi_{\mathrm{B}} / \Phi_{0}} .
$$

In the definition of $\Lambda$, the parameter $L$ is the length of a single arm of the interferometer (the two arms are chosen of equal lengths). If the parameter $\Lambda$ vanishes, the SO interaction simply disappears and we are led to the Abelian $\mathrm{AB}$ situation. If $\cos \Lambda=0$, the operator $\mathcal{U}_{a}$ is diagonal in the input spinor basis, which means that a perfect spin filtering is possible in this original basis if one of the two eigenvalues $\lambda_{ \pm}^{a}$ vanishes (in which case the corresponding component is filtered and only the other component survives in the detector $a$ ). If it is not the case, filtering may still be possible, but in a different basis, tilted from the original one [29].

The traceless condition simplifies the diagonalization of $\mathbf{I M}_{2 \times 2}$, and the eigenvalues for $\mathcal{U}_{a}$ are easily found to be

$$
\lambda_{ \pm}^{a}=\mathcal{A}_{+}\left[\cos ^{2} \Lambda-\sin ^{2} \Lambda \sin 2 \theta\right] \mp \mathrm{i} \sin \Lambda \sqrt{\mathcal{A}_{-}^{2} \sin ^{2} \Lambda \cos ^{2} 2 \theta+2 \mathcal{A}_{+}^{2} \cos ^{2} \Lambda(1+\sin 2 \theta)} .
$$

The filtering condition (e. g. of the + component of the spinor) is guaranteed by the condition $\lambda_{a}^{+}=0$. It can be shown that this condition can always be fulfilled by a convenient choice of the magnetic flux between the arms of the interferometer. As we have discussed above, an interesting feature is that, depending on the value of $\Lambda$ (remember that the Rashba amplitude can be tuned experimentally), it is possible to achieve this condition in a non-tilted basis. When the perfect filtering condition is satisfied, the amplitude of the non-filtered component is obtained via the other eigenvalue, $\lambda_{a}^{-} \Psi_{0}^{-}$. In the following figures we propose illustrations in the most generic case, i. e. the tilted basis, since this is the situation that occurs in general. In contrast, the conditions for perfect filtering in the original basis requires a particular relation between the Rashba and Dresselhaus amplitudes through the relation $\cos \Lambda=0$. We thus show contour plots of the magnetic flux which allows for perfect filtering and for the intensity of the non-filtered component in the detector in the tilted basis, in the plane $\alpha, \beta$. 


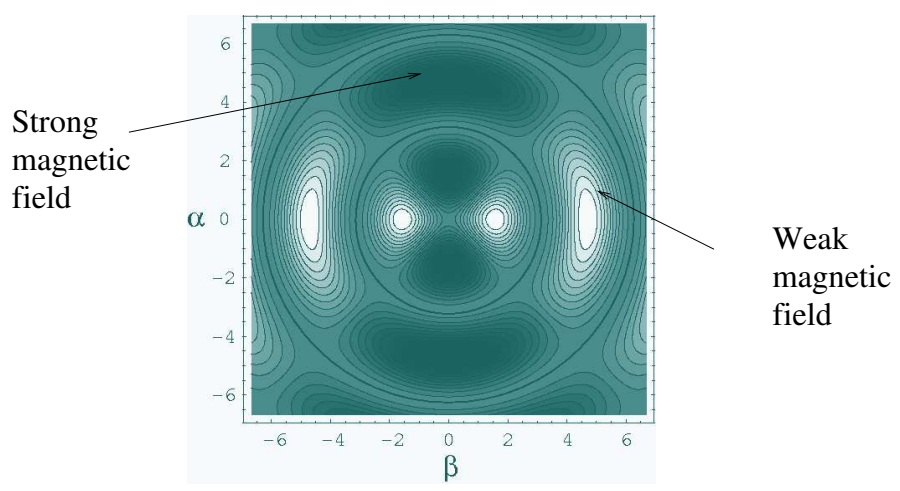

Figure 2. Perfect filtering by interference for the tilted axis. The plot shows a contour plot of $\sin \pi \Phi_{\mathrm{B}} / \Phi_{0}$ in the plane $\alpha, \beta$ (in units of $\hbar /\left(m^{*} L\right)$ ). The darker regions indicate larger values for the magnetic flux needed to yield the condition of perfect filtering, from an unpolarized input. Highlighted circle and diagonal lines depict the zero flux solutions that yield perfect filtering.

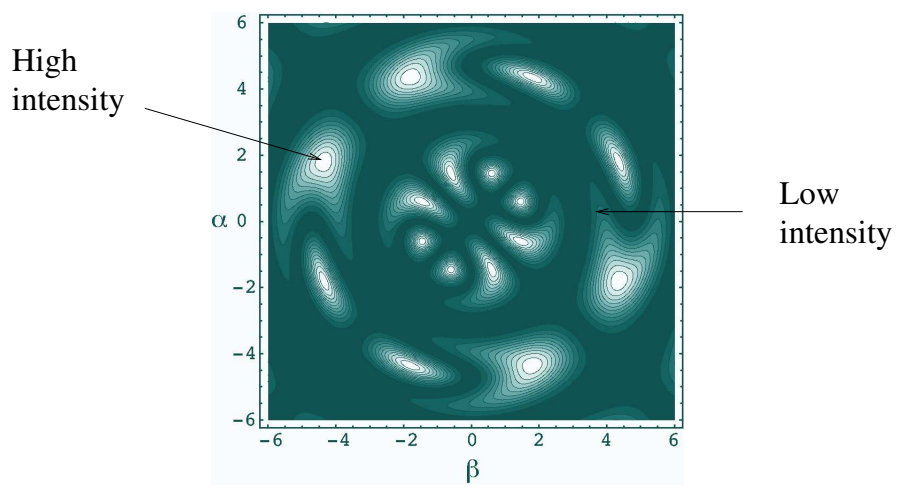

Figure 3. Perfect filtering probability for the tilted axis. The figure shows a contour plot (in the plane $\alpha, \beta$ in units of $\hbar /\left(m^{*} L\right)$ ) of the intensity in detector $a,\left|\Psi_{a}^{-}\right|^{2}$, assuming $\left|\Psi_{0}^{-}\right|=1$. Perfect spin filtering is satisfied by the condition $\lambda_{a}^{+}=0$. The lighter regions indicate larger values for the intensity of filtering for the relation between parameters depicted in figure 2 . Note that circles and diagonals evident from figure 2 correspond to zero output amplitude.

\section{Conclusion}

We have presented a non-Abelian gauge theory suited to deal with non-relativistic quantum mechanics in the presence of various types of spin-orbit interactions. This formulation has the advantage of a correct definition of the spin current density and is adapted to treat problems like topological quantization and spin filtering. This is a very elegant way to study spintronics.

\section{References}

1. Ryder L.H. Quantum Field Theory. Cambridge University Press, 1985.

2. Rebei A., Heinonen O., Phys. Rev. B, 2006, 73, 153306.

3. Jin P.Q., Li Y.Q., Zhang F.C., J. Phys. A: Math. Gen, 2006, 39, 7115.

4. Leurs B.W.A., Nazarioa Z., Santiagoa D.I., Zaanen J., Ann. Phys., 2008, 323, 907.

5. Medina E., López A., Berche B., Europhys. Lett., 2008, 83, 47005.

6. Goldhaber A.S., Phys. Rev. Lett., 1989, 62, 482.

7. Mineev V.P., Volovik G.E., J. Low Temp. Phys., 1992, 89, 823.

8. Frohlich J., Studer U.M., Rev. Mod. Phys., 1993, 65, 733.

9. Frohlich J., Studer U.M., Commun. Math. Phys., 1992, 148, 552.

10. Tokatly I.V., Phys. Rev. Lett., 2008, 101, 106601. 
11. Rashba R.E., Sov. Phys. Sol. State, 1960, 2, 1109.

12. Dresselhaus G., Phys. Rev., 1955, 100, 580.

13. Winkler R., Spin-Orbit Coupling Effects in Two Dimensional Electron and Hole Systems. Springer, 2003.

14. Nitta J., Koga T., J. Supercond., 2003, 16, 689.

15. Zutic I., Fabian J., Das Sarma S., Rev. Mod. Phys., 2004, 76, 323.

16. Usaj G., Balseiro C.A., Europhys. Lett., 2005, 72, 631.

17. Engel H.A., Rashba E.I., Halperin B.I. Preprint arXiv: cond-mat/0603306.

18. Jin P.Q., Li Y.Q., Zhang F.C., J. Phys. Math Gen., 2006, 35, 7115.

19. Weinberg S. The Quantum Theory of Fields, Vol. II. Cambridge University Press, 1996;Peskin M., Shroeder D. An Introduction to Quantum Field Theory. Addison-Wesley, 1996.

20. Hughues R.J., Nucl. Phys. B, 1978, 161, 156.

21. Rashba E.I., Phys. Rev. B, 2003, 68, 241315(R).

22. López A., Medina E., Bolívar N., Berche B. Preprint arXiv:0902.4635.

23. Tinkham M. Introduction to Superconductivity. Mac Graw Hill, 1996.

24. Rashba E.I., Phys. Rev. B, 2004, 70, 201309.

25. Wu T.T., Yang C.N., Phys. Rev. D, 1975, 12, 3845.

26. Aharonov Y., Casher A., Phys. Rev. Lett., 1984, 53, 319.

27. Ji Y., Chung Y., Sprinzak D., Heiblum M., Mahalu D., Shtrikman H., Nature, 2003, 422, 415.

28. López A., Medina E., Bolívar N., Berche B. Preprint arXiv:0903.2468.

29. Hatano N., Shirasaki R., Nakamura H., Phys. Rev. A, 2007, 75, 032107.

30. Chen S.-H., Chang C.-R., Phys. Rev. B, 2008, 77, 045324.

31. Feve G., Oliver W.D., Aranzana M., Yamamoto Y., Phys. Rev. B, 2002, 66, 155328.

\title{
Підхід теорії калібрувальних полів до вивчення спінового переносу в двовимірному електронному газі
}

\author{
Б. Берш ${ }^{1,2}$, Н. Болівар ${ }^{3}$, А. Лопес ${ }^{2}$, Е. Медіна ${ }^{1,2}$ \\ 1 Група статистичної фізики, Відділ Р2М, Інститут ім. Жана Ламора, F-54506 Нансі, Франція \\ 2 Центр фізики, Венесуельський інститут наукових досліджень, 1020А Каракас, Венесуела \\ 3 Фізичний факультет, Центральний університет Венесуели, Каракас, Венесуела
}

Отримано 19 червня 2009 р.

\begin{abstract}
В рамках інтерпретації $U(1) \times S U(2)$ калібрувальної теорії обговорюється гамільтоніан Паулі, що включає спін-орбітальну взаємодію, де калібрувальна симетрія порушується. Така інтерпретація відкриває можливість проникнення в суть проблеми спінових потоків в конденсованому середовищі і може бути узагальнена на випадок спін-орбітальної взаємодії Рашби та Дрессельхауса. Представлено кілька висновків, що слідують з такого формулювання: і) воно автоматично приводить до нульової спінової провідності на відміну від передбачень калібрувально-симетричного підходу; іi) умова топологічного квантування приводить до квантування напруги; iii) можна запропонувати спінові інтерферометри, в яких, починаючи з довільного вхідного неполяризованого спінора, завжди можна утворити ідеальні умови спінового фільтрування.
\end{abstract}

Ключові слова: спін-орбітальна взаємодія, теорія калібрувальних полів, спіновий перенос, спіновий ефект Голла

PACS: $75.25 .+z, 85.75 .-d, 03.65 . V f$ 